\title{
Efecto de la aplicación aguda de fluoxetina sobre una tarea de miedo condicionado al contexto en ratas sometidas a restricción comportamental $^{*}$
}

\section{Effect of Acute Fluoxetine Application on a Context Fear Conditioned Task in Behaviorally Restrained Rats}

Recibido: febrero14 de 2010 | Revisado: abril 2 de 2010 | Aceptado: mayo 15 de 2010

\author{
LAURA A. LEÓN ** \\ RODRIGO SIERRA ${ }^{* * * *}$ \\ LAURA P. NitOlA \\ FERNANDO P. CÁRDENAS \\ Universidad de los Andes, Bogotá, Colombia \\ JUAN C. URAZÁN ${ }^{* * * *}$ \\ Universidad Surcolombiana de Neiva, Colombia \\ LINA T. LIZARASO ****** \\ Universidad de los Andes, Bogotá, Colombia
}

Para citar este artículo. León, L.A., Sierra, R., Nitola, L.P., Cárdenas F., P., Urazán, J.C., \& Lizaraso, L.T. (2010). Efecto de la aplicación aguda de fluoxetina sobre una tarea de miedo condicionado al contexto en ratas sometidas a restricción comportamental. Universitas Psychologica, 9 (3), 689-696.

* Esta investigación fue financiada por el proyecto FAPA de la Universidad de los Andes, Bogotá. La Facultad de Salud de la Universidad Surcolombiana de Neiva apoyó económicamente a Juan C. Urazán. Dirigir correspondencia a Laboratorio de Neurociencia y Comportamiento, Departamento de Psicología, Universidad de los Andes, Cra 1 \#18A-12, Bogotá, Colombia. Tel: + 571 3394999-3624; Fax +5713394999-2597.E-mail: lucarden@uniandes. edu.co.

*** Laboratorio de Neurociencia y Comportamiento, Departamento de Psicología, Universidad de los Andes, Cra 1 \#18A-12, Bogotá, Colombia. Tel: +5713394999-3624.Correo electrónico:la-leon@ uniandes.edu.co

*** Laboratorio de Neurociencia y Comportamiento. E-mail: neuro@uniandes.edu.co; lucarden@uniandes.edu.co

${ }^{* * * * *}$ Facultad de Salud, programa de Psicología. E-mail: neuro@uniandes.edu.co

******* Facultad de Ciencias, Laboratorio de Neurociencia y Comportamiento. E-mail: neuro@uniandes. edu.co.

\section{RES UMEN}

Para estudiar el efecto del aumento comportamental o farmacológico de la ansiedad sobre la adquisición del miedo condicionado al contexto, 32 ratas Wistar (275 $\pm 25 \mathrm{gm})$ divididas en dos grupos (restricción comportamental y control) recibieron fluoxetina (ig, $4 \mathrm{mg} / \mathrm{kg} ; 1 \mathrm{ml}$ ) o solución salina (ig, 0.9\%). Luego fueron entrenadas en una tarea de miedo condicionado al contexto. El ANOVA de dos vías mostró diferencias significativas para el factor tratamiento $\left(F_{[1,28]}=25.261 ; P<0.001\right)$. Los sujetos tratados con fluoxetina presentaron menor tiempo de congelamiento (Student Newman-Keuls; $P<0.05)$. No hubo diferencias significativas para la restricción, ni para la interacción entre factores $\left(F_{[1,28]}=0.115 ; P=0.737\right.$ y $F_{[1,28]}=0.016$; $P=0.899)$. Así, la restricción no alteró la adquisición del miedo condicionado indicando que el aumento de liberación de 5-HT así inducido, no es comparable al inducido por fluoxetina. La fluoxetina deterioró la adquisición de la respuesta de miedo, indicando que el mecanismo por el cual la ansiedad interrumpe el aprendizaje puede ser serotoninérgico.

Palabras clave autores

Fluoxetina, memoria emocional, miedo condicionado, serotonina, rata Palabras clave descriptor

Fluoxetina, serotonina, ratas, psicología, miedo, efectos de drogas.

\section{A B S T R A C T}

In order to study the effect of behavioral or pharmacologically enhanced anxiety on the acquisition of contextual fear conditioning, thirty two Wistar rats $(275 \pm 25 \mathrm{gm})$ were divided in two groups (behavioral restriction and control). Half of each group received saline solution (ig.; 0.9\%) or fluoxetine (ig.; $4 \mathrm{mg} / \mathrm{Kg}$ ) before the fear conditioning procedure. The two way ANOVA showed significant differences for treatment $\left(F_{[1,28]}=25.261 ; P<0.001\right)$. Student Newman-Keuls showed that subjects treated with fluoxetine had 
lower freezing times. There were no significant differences nor for restriction neither for the interaction between the factors $\left(F_{[1,28]}=0.115 ; P=0.737\right.$ y $\left.F_{[1,28]}=0.016 ; P=0.899\right)$. Thus, the restriction procedure used did not modify the acquisition of the conditioned fear response suggesting that the putative 5-HT enhancement induced is not comparable to that induced by fluoxetine. Acute fluoxetine disrupted the acquisition of the conditioned fear response, suggesting that the mechanism by means of which anxiety disrupts learning could be serotonergic in nature.

Keywords authors

Fluoxetine; Emotional Memory; Conditioned Fear; Serotonin; Rat Keywords plus

Fluoxetine, Serotonin, Rats, Psychology, Fear, Drug Effects.

\section{Introducción}

Existe una clara relación entre el aumento agudo de la disponibilidad de 5-HT extracelular y el aumento de los niveles de ansiedad (Alves, Pinheiro, Motta, Landeira-Fernández \& Cruz, 2004; Andrade \& Graeff, 2001; Bertrand, Lehmann, Lazarus, Jeltsch \& Cassel, 2000; Borelli, Gargaro, dos Santos \& Brandao, 2005; Graeff, 2002; Graeff, Viana \& Mora, 1997; Micheau \& Van Marrewijk, 1999; Moraes, Bertoglio \& Carobrez, 2008; Pinheiro, Zangrossi, Jr., Del Ben \& Graeff, 2007).

El sistema serotoninérgico está implicado en la regulación de actividades de sueño, humor, impulso sexual y funcionamiento cognoscitivo, entre otras (El Mallakh \& Abraham, 2007; Harmer, 2008; Strobel et al., 2007; Terry, Jr., Buccafusco \& Wilson, 2008; Thomasius et al., 2006). El origen del sistema serotoninérgico cerebral son las neuronas de los núcleos de rafé, cuyas proyecciones llegan a casi todas las demás regiones encefálicas, siendo locus coeruleus, hipocampo, amígdala y córtex frontal quizá las más importantes en su papel regulador de la ansiedad (dos Santos, de Andrade \& Zangrossi, 2008; Holmes, 2008; Molodtsova, 2008; Pum, Huston \& Muller, 2009). Uno de los problemas comunes de la aproximación farmacológica al tratamiento de la depresión, con inhibidores selectivos de la recaptación de 5-HT (SSRIs) es, justamente, el aumento inicial de los niveles basales de ansiedad (Dawson \& Bromidge, 2008; Salchner \& Singewald, 2002).

Si bien las drogas más potentes para el control de los estados de ansiedad elevada, actúan sobre el sistema GABAérgico (benzodiazepinas y barbitúricos), algunos compuestos de acción regulatoria sobre los sistemas serotoninérgicos son preferidos por su ausencia (al menos aparente) de efectos secundarios o de alteraciones a largo plazo, tales como hipnosis, sedación o dependencia. Dentro de ellos, se cuentan agonistas selectivos de receptores 5- $\mathrm{HT}_{1 \mathrm{~A}}$, tales como la buspirona e incluso la utilización de dosis elevadas de SSRIs (de Paula Soares \& Zangrossi, Jr., 2009; De Vry, Schreiber, Melon, Dalmus \& Jentzsch, 2004; Ettenberg \& Bernardi, 2006; Harada et al., 2006; Lim et al., 2008; Panzer, 2005; Taylor \& Stein, 2006).

Por otro lado, ha sido reportado que la exposición de animales a estímulos estresores agudos e incontrolables ocasiona aumento de los niveles plasmáticos y extracelulares de 5-HT y respuestas comportamentales de ansiedad como el congelamiento (Christianson et al., 2010; Maier \& Watkins, 2005; Strong, Greenwood \& Fleshner, 2009). El aumento de la ansiedad posee conocidos efectos deletéreos sobre la adquisición y evocación de memorias y recuerdos no emocionales (Schwabe, Bohringer \& Wolf, 2009; Schwabe \& Wolf, 2010). La regulación noradrenérgica, ejercida por 5-HT, podría explicar algunos de estos efectos a corto plazo (Sodero, Valdomero, Cuadra, Ramírez \& Orsingher, 2004); a largo plazo, el aumento de la actividad en el eje hioptálamo-hipófisis-adrenal, ha sido propuesto como explicación del fenómeno (Leonard, 2005).

En este trabajo, se estudió el efecto del aumento de la ansiedad mediante dos procedimientos diferentes: uno farmacológico (aplicación aguda de fluoxetina) y uno comportamental (exposición a un protocolo de restricción comportamental), sobre la adquisición de memorias emocionales, en sujetos con diferentes niveles de estrés preentrenamiento. 


\section{Materiales y métodos}

\section{Animales}

Se utilizaron treinta y dos ratas Wistar hembra, ingenuas $(275 \pm 25 \mathrm{~g})$, cicladas previamente con estradiol. Todos los animales fueron obtenidos del bioterio de la Universidad de los Andes. Los sujetos fueron alojados en grupos de cuatro, en cajas de polipropileno $(43 \times 31 \times 18 \mathrm{~cm})$ y mantenidos baja un ciclo claro-oscuro (12:12, con la luz encendida a las 07:00 h). Los animales tuvieron acceso libre a comida y agua durante todo el experimento. Luego de la llegada de los animales al laboratorio, se permitió un periodo de tres días para la familiarización con las condiciones del mismo. Todas las ratas recibieron tres minutos diarios de manipulación, por cinco días antes del inicio de los experimentos. Todos los protocolos experimentales utilizados en este trabajo se realizaron de acuerdo con las normas éticas recomendadas por las normas colombianas para el cuidado de animales de Laboratorio (leyes 84 de 1989 y 8430 de 1993, que a su vez están basadas en las normas de los National Institutes of Health Guide for Care and Use of Laboratory Animals de Estados Unidos (No. 86-23, revisada en1985).

\section{Drogas}

Se utilizó fluoxetina (Medley, Brasil) en una concentración de $4 \mathrm{mg} / \mathrm{kg}$, disuelta en solución salina (0.9\%). Los sujetos del grupo control recibieron solución salina $(0.9 \%)$.

\section{Aparatos}

Se utilizó una caja de condicionamiento de miedo al contexto. Brevemente descrito, se trata de una caja con paredes en madera, $(40 \times 40 \times 40 \mathrm{~cm})$ y piso de rejilla metálica, iluminada por luz ambiental de aproximadamente 60 lux en el centro. El comportamiento del animal dentro de la caja era registrado por medio de una cámara y almacenado digitalmente, para su posterior análisis. El piso de la caja estaba conectado a un generador de choque (MM-01), controlado por un computador.
Para la aplicación intragástrica del compuesto, se utilizaron jeringas estándar de administración intragástrica.

\section{Procedimiento}

\section{Restricción}

El experimento inició con la distribución aleatoria de los sujetos a uno de dos grupos de restricción (Res[-] Res[+]). Los sujetos del grupo Res[+] fueron inmovilizados comportamentalmente durante veinte minutos, utilizando cilindros estándar de inmovilización $(7 \mathrm{~cm}$ de diámetro $\mathrm{x} 18 \mathrm{~cm}$ de longitud). Los sujetos del grupo Res[-] permanecieron en sus cajas hábitat. Terminado el período de restricción, la mitad de los sujetos de cada grupo recibió la administración intragástrica de un mililitro de fluoxetina $(4 \mathrm{mg} / \mathrm{kg}$ ) o de solución salina $(0.9 \%)$ y fueron colocados nuevamente en sus respectivas cajas durante treinta minutos, tiempo adecuado para que el aumento de los niveles de 5-HT intracerebral alcanzara su pico (Silva \& Brandao, 2000).

\section{Condicionamiento del miedo}

Terminado el período de treinta minutos, los sujetos eran transportados a la caja de condicionamiento contextual de miedo. El protocolo de condicionamiento de miedo consistió en una variación al descrito por Landeira-Fernández, DeCola, Kim $\&$ Fanselow (2006). Cada sujeto era colocado por primera vez en la caja de condicionamiento por ocho minutos (sesión de adquisición del condicionamiento). Luego de trascurridos los primeros cinco minutos y sin ningún evento de señalización, se aplicó un único choque de $0.5 \mathrm{~mA}$ y 1 segundo de duración. Terminado el periodo de ocho minutos, el sujeto era llevado a su respectiva caja hábitat. Veinticuatro horas después, se realizaba la sesión de test del condicionamiento. La sesión de test consistía en la colocación del animal nuevamente en la misma caja de condicionamiento, durante cinco minutos. En esta sesión no se administró ningún estímulo aversivo. En esta sesión se realizaba el 
conteo de tiempo de congelamiento durante todo el tiempo de permanencia del sujeto, para lo cual, a cada dos segundos se evaluaba y registraba, por parte de un observador entrenado, el estado de congelamiento del animal, utilizando el programa Time Sampling. El congelamiento fue definido como la ausencia total de movimientos del cuerpo, cabeza y vibrisas, con la excepción de los necesarios para respirar (Landeira-Fernández et al., 2006).

\section{Análisis estadístico}

Los datos correspondientes al tiempo de congelamiento en la sesión de test fueron analizados utilizando un análisis de varianza (ANOVA) de dos vías, siendo restricción el primer factor y tratamiento, el segundo. Cuando fue necesario, las diferencias en los promedios de los grupos fueron comparadas utilizando el test post hoc de Student Newman Keuls. El alfa fue establecido en 0.05.

\section{Resultados}

La Figura 1 muestra el tiempo de congelamiento en la sesión de test para todos los grupos. El ANO. VA mostró diferencias significativas para el factor tratamiento $\left(F_{[1,28]}=25.261 ; P<0.001\right)$. La comparación post hoc (Student Newman-Keuls) de los promedios de los grupos mostró que los sujetos que recibieron fluoxetina, tuvieron menores tiempo de congelamiento que los que recibieron solución salina $(P<0.05)$.

El ANOVA no mostró diferencias significativas para el factor restricción $\left(F_{[1,28]}=0.115 ; P=\right.$ 0.737). Tampoco fueron halladas diferencias significativas para la interacción de los factores $\left(F_{[1,28]}\right.$ $=0.016 ; P=0.899$ ).

\section{Discusión}

Los resultados obtenidos muestran que la administración intragástrica de fluoxetina disminuye el tiempo de congelamiento en la sesión de test, indicando claramente una interferencia con la formación del condicionamiento de miedo al contexto. El efecto hallado para la fluoxetina fue inde-

\section{Figura 1}

Tiempo de congelamiento para los diferentes grupos en la sesión de test

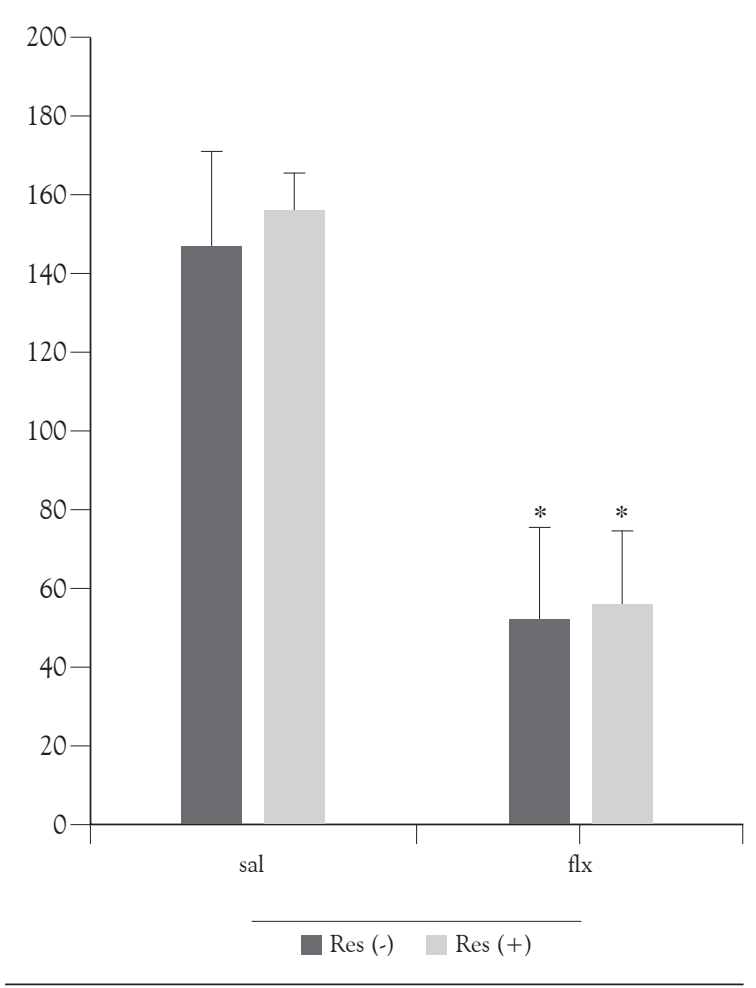

Fuente: elaboración propia.

pendiente de la previa exposición al protocolo de restricción. De hecho, en los sujetos que recibieron solución salina no se observaron diferencias relacionadas con la previa exposición al protocolo de restricción comportamental.

Se ha reportado, en la literatura, que la aplicación de SSRIs conlleva de forma aguda al aumento de la liberación de 5-HT que conduce a su vez al aumento de los niveles de ansiedad (Drapier et al., 2007; Greenwood, Strong, Brooks \& Fleshner, 2008). Alteraciones en el sentido opuesto, es decir, disminución de los niveles de 5-HT ha sido correlacionada con efectos ansiolíticos, entre ellos vale la pena mencionar reportes de lesión de los núcleos de rafé medial (Andrade, Macedo, Zangrossi, Jr. \& Graeff, 2004; Netto, Silveira, Coimbra, Joca \& Guimaraes, 2002).

La relación entre niveles de 5-HT y formación de memorias emocionales ha sido estudiada utili- 
zando condicionamientos aversivos particulares. Reportes de LeDoux y colaboradores (Burghardt, Sullivan, McEwen, Gorman \& LeDoux, 2004) indican que la administración aguda de SSRIs mejora la adquisición de miedo condicionado a un tono. De acuerdo a estos reportes, cabría esperar que el aumento de 5-HT, bien por la aplicación de fluoxetina como por la exposición al protocolo de restricción comportamental, pudiera mejorar el aprendizaje de la respuesta de miedo condicionado; sin embargo, nuestros datos no apoyan esta suposición. Una posible explicación sería que en el caso del miedo condicionado a tonos, la amígdala, se sabe, es la estructura más implicada (Doyere, Debiec, Monfils, Schafe \& LeDoux, 2007; Johnson et al., 2008; Lamprecht, Dracheva, Assoun \& LeDoux, 2009), mientras que en nuestro caso, la utilización del contexto como estímulo condicionado hace que sea el hipocampo y no la amígdala, la estructura más implicada (Alves et al., 2004; Silva, Gargaro \& Brandao, 2004).

Estudios realizados sobre el papel del hipocampo en la regulación de los efectos emocionales de 5-HT indican que la región dorsal está principalmente relacionada con la mediación de efectos de tipo ansiolítico (Carvalho, Masson, Brandao \& Souza Silva, 2008; dos Santos, de Andrade \& Zangrossi, 2008). La región ventral resulta crucial para la expresión de las respuestas de ansiedad (posiblemente a través de sus conexiones con la corteza prefrontal) y para la evocación de los recuerdos emocionales (Adhikari, Topiwala, \& Gordon, 2010; Ohmura et al., en prensa). De esta forma, es posible que la participación de diversas áreas límbicas sea diferencial, dependiendo de si se trata de un condicionamiento ante un estímulo particular o no (Cruz-Morales et al., 2008; Herry et al., 2008; Maren, 2008). Un ejemplo análogo de esta situación es la disociación de los efectos de 5-HT sobre evitación y escape (Viana, Zangrossi, Jr. \& Onusic, 2008). Nuestros resultados demuestran la interferencia del aumento de 5-HT en la adquisición del condicionamiento de miedo al contexto, pero no es posible, a partir de ellos, determinar algún efecto sobre la evocación de la situación aversiva.
Resulta de interés mencionar reportes acerca del papel del ejercicio en la inhibición de la aparición de los efectos ansiogénicos inducidos por fluoxetina aguda en ratas Fischer 344 (Greenwood et al., 2008). En nuestro caso, la restricción comportamental fue utilizada para aumentar endógenamente los niveles de 5-HT. Debido a que la restricción comportamental puede ocasionar un subsecuente aumento de actividad locomotriz, es posible que el periodo en que los sujetos permanecieron en sus jaulas, antes del entrenamiento en la tarea de miedo condicionado, haya facilitado un aumento de la actividad locomotriz, equiparable al ejercicio descrito en los trabajos de Greenwood y colaboradores. Sería posible entonces que la perturbación en la adquisición del condicionamiento de miedo al contexto, visto en los sujetos tratados con fluoxetina pueda haber sido debido al efecto inhibitorio de la actividad física sobre la ansiedad serotoninérgica, pues es un efecto que, al parecer, está presente en varias cepas de ratas (Greenwood, Strong, Foley \& Fleshner, 2009; Greenwood \& Fleshner, 2008; Greenwood, Foley, Burhans, Maier $\&$ Fleshner, 2005; Greenwood et al., 2003).

\section{Referencias}

Adhikari, A., Topiwala, M. A. \& Gordon, J. A. (2010). Synchronized activity between the ventral hippocampus and the medial prefrontal cortex during anxiety. Neuron, 65, 257-269.

Alves, S. H., Pinheiro, G., Motta, V., Landeira-Fernandez, J. \& Cruz, A. P. (2004). Anxiogenic effects in the rat elevated plus-maze of 5-HT(2C) agonists into ventral but not dorsal hippocampus. Behavioral Pharmacology, 15, 37-43.

Andrade, T. G. \& Graeff, F. G. (2001). Effect of electrolytic and neurotoxic lesions of the median raphe nucleus on anxiety and stress. Pharmacology, Biochemistry and Behavior, 70, 1-14.

Andrade, T. G., Macedo, C. E., Zangrossi, H., Jr. \& Graeff, F. G. (2004). Anxiolytic-like effects of median raphe nucleus lesion in the elevated T-maze. Behavioural Brain Research, 153, 55-60.

Bertrand, F., Lehmann, O., Lazarus, C., Jeltsch, H. \& Cassel, J. C. (2000). Intraseptal infusions of 8-OH- 
DPAT in the rat impairs water-maze performances: Effects on memory or anxiety? Neuroscience Letters, 279, 45-48.

Borelli, K. G., Gargaro, A. C., dos Santos, J. M. \& Brandao, M. L. (2005). Effects of inactivation of serotonergic neurons of the median raphe nucleus on learning and performance of contextual fear conditioning. Neuroscience Letters, 387, 105-110.

Burghardt, N. S., Sullivan, G. M., McEwen, B. S., Gorman, J. M. \& LeDoux, J. E. (2004). The selective serotonin reuptake inhibitor citalopram increases fear after acute treatment but reduces fear with chronic treatment: A comparison with tianeptine. Biological Psychiatry, 55, 1171-1178.

Carvalho, M. C., Masson, S., Brandao, M. L. \& Souza Silva, M. A. (2008). Anxiolytic-like effects of substance P administration into the dorsal, but not ventral, hippocampus and its influence on serotonin. Peptides, 29, 1191-1200.

Christianson, J. P., Ragole, T., Amat, J., Greenwood, B. N., Strong, P. V., Paul, E. D. et al. (2010). 5-hydroxytryptamine $2 \mathrm{C}$ receptors in the basolateral amygdala are involved in the expression of anxiety after uncontrollable traumatic stress. Biological Psychiatry, 67, 339-345.

Cruz-Morales, S. E., García-Saldivar, N. L., GonzálezLópez, M. R., Castillo-Roberto, G., Monroy, J. \& Domínguez, R. (2008). Acute restriction impairs memory in the elevated T-maze (ETM) and modifies serotonergic activity in the dorsolateral striatum. Behavioural Brain Research, 195, 187-191.

Dawson, L. A. \& Bromidge, S. M. (2008). 5-HT1 receptor augmentation strategies as enhanced efficacy: Therapeutics for psychiatric disorders. Current Topics in Medical Chemistry, 8, 1008-1023.

de Paula Soares, V. \& Zangrossi, H., Jr. (2009). Stimulation of 5-HT1A or 5-HT2A receptors in the ventrolateral periaqueductal gray causes anxiolytic-, but not panicolytic-like effect in rats. Behavioural Brain Research, 197, 178-185.

De Vry, J., Schreiber, R., Melon, C., Dalmus, M. \& Jentzsch, K. R. (2004). 5-HT1A receptors are differentially involved in the anxiolytic- and antidepressant-like effects of 8-OH-DPAT and fluoxetine in the rat. European Neuropsychopharmacology, 14, 487-495. dos Santos, L., de Andrade, T. G. \& Zangrossi, J. H. (2008). 5-HT1A receptors in the dorsal hippocampus mediate the anxiogenic effect induced by the stimulation of 5-HT neurons in the median raphe nucleus. European Neuropsychopharmacology, 18, 286-294.

Doyere, V., Debiec, J., Monfils, M. H., Schafe, G. E. \& LeDoux, J. E. (2007). Synapse-specific reconsolidation of distinct fear memories in the lateral amygdala. Nature Neuroscience, 10, 414-416.

Drapier, D., Bentue-Ferrer, D., Laviolle, B., Millet, B., Allain, H., Bourin, M. et al. (2007). Effects of acute fluoxetine, paroxetine and desipramine on rats tested on the elevated plus-maze. Behavioural Brain Research, 176, 202-209.

El Mallakh, R. S. \& Abraham, H. D. (2007). MDMA (Ecstasy). Annals in Clinical Psychiatry, 19, 45-52.

Ettenberg, A. \& Bernardi, R. E. (2006). Anxiolytic-like actions of buspirone in a runway model of intravenous cocaine self-administration. Pharmacology, Biochemistry and Behavior, 85, 393-399.

Graeff, F. G. (2002). On serotonin and experimental anxiety. Psychopharmacology (Berl), 163, 467-476.

Graeff, F. G., Viana, M. B. \& Mora, P. O. (1997). Dual role of 5-HT in defense and anxiety. Neuroscience and Biobehavioral Reviews, 21, 791-799.

Greenwood, B. N. \& Fleshner, M. (2008). Exercise, learned helplessness, and the stress-resistant brain. Neuromolecular Medicine, 10, 81-98.

Greenwood, B. N., Foley, T. E., Burhans, D., Maier, S. F. $\&$ Fleshner, M. (2005). The consequences of uncontrollable stress are sensitive to duration of prior wheel running. Brain Research, 1033, 164-178.

Greenwood, B. N., Foley, T. E., Day, H. E., Campisi, J., Hammack, S. H., Campeau, S. et al. (2003). Freewheel running prevents learned helplessness/ behavioral depression: Role of dorsal raphe serotonergic neurons. The Journal of Neuroscience, 23, 2889-2898.

Greenwood, B. N., Strong, P. V., Brooks, L. \& Fleshner, M. (2008). Anxiety-like behaviors produced by acute fluoxetine administration in male Fischer 344 rats are prevented by prior exercise. Psychopharmacology (Berl), 199, 209-222.

Greenwood, B. N., Strong, P. V., Foley, T. E. \& Fleshner, M. (2009). A behavioral analysis of the impact 
of voluntary physical activity on hippocampusdependent contextual conditioning. Hippocampus, 19, 988-1001.

Harada, K., Aota, M., Inoue, T., Matsuda, R., Mihara, T., Yamaji, T. et al. (2006). Anxiolytic activity of a novel potent serotonin 5-HT2C receptor antagonist FR260010: A comparison with diazepam and buspirone. European Journal of Pharmacology, 553, 171-184.

Harmer, C. J. (2008). Serotonin and emotional processing: Does it help explain antidepressant drug action? Neuropharmacology, 55, 1023-1028.

Herry, C., Ciocchi, S., Senn, V., Demmou, L., Muller, C. \& Luthi, A. (2008). Switching on and off fear by distinct neuronal circuits. Nature, 454, 600-606.

Holmes, A. (2008). Genetic variation in cortico-amygdala serotonin function and risk for stress-related disease. Neuroscience and Biobehavioral Reviews, 32, 1293-1314.

Johnson, L. R., Hou, M., Ponce-Álvarez, A., Gribelyuk, L. M., Alphs, H. H., Albert, L. et al. (2008). A recurrent network in the lateral amygdala: A mechanism for coincidence detection. Front Neural Circuits, 2, 3.

Lamprecht, R., Dracheva, S., Assoun, S. \& LeDoux, J. E. (2009). Fear conditioning induces distinct patterns of gene expression in lateral amygdala. Genes, Brain and Behavior, 8, 735-743.

Landeira-Fernández, J., DeCola, J. P., Kim, J. J. \& Fanselow, M. S. (2006). Immediate shock deficit in fear conditioning: Effects of shock manipulations. Behavioral Neuroscience, 120, 873-879.

Leonard, B. E. (2005). The HPA and immune axes in stress: The involvement of the serotonergic system. European Psychiatry, 20 (Suppl. 3), S302S306.

Lim, L. W., Temel, Y., Sesia, T., Vlamings, R., VisserVandewalle, V., Steinbusch, H. W. et al. (2008). Buspirone induced acute and chronic changes of neural activation in the periaqueductal gray of rats. Neuroscience, 155, 164-173.

Maier, S. F. \& Watkins, L. R. (2005). Stressor controllability and learned helplessness: The roles of the dorsal raphe nucleus, serotonin, and corticotropin-releasing factor. Neuroscience and Biobehavioral Reviews, 29, 829-841.
Maren, S. (2008). Pavlovian fear conditioning as a behavioral assay for hippocampus and amygdala function: Cautions and caveats. European Journal of Neuroscience, 28, 1661-1666.

Micheau, J. \& Van Marrewijk, B. (1999). Stimulation of 5-HT1A receptors by systemic or medial septum injection induces anxiogenic-like effects and facilitates acquisition of a spatial discrimination task in mice. Progress in Neuropsychopharmacology and Biological Psychiatry, 23, 1113-1133.

Molodtsova, G. F. (2008). Serotonergic mechanisms of memory trace retrieval. Behavioural Brain Research, 195, 7-16.

Moraes, C. L., Bertoglio, L. J. \& Carobrez, A. P. (2008). Interplay between glutamate and serotonin within the dorsal periaqueductal gray modulates anxiety-related behavior of rats exposed to the elevated plus-maze. Behavioural Brain Research, 194, 181-186.

Netto, S. M., Silveira, R., Coimbra, N. C., Joca, S. R. \& Guimaraes, F. S. (2002). Anxiogenic effect of median raphe nucleus lesion in stressed rats. Progress in Neuropsychopharmacology and Biological Psychiatry, 26, 1135-1141.

Ohmura, Y., Izumi, T., Yamaguchi, T., Tsutsui-Kimura, I., Yoshida, T. \& Yoshioka, M. (en prensa). The Serotonergic Projection from the Median Raphe Nucleus to the Ventral Hippocampus is Involved in the Retrieval of Fear Memory Through the Corticotropin-Releasing Factor Type 2 Receptor. Neuropsychopharmacology.

Panzer, M. J. (2005). Are SSRIs really more effective for anxious depression? Annals in Clinical Psychiatry, 17, 23-29.

Pinheiro, S. H., Zangrossi, H., Jr., Del Ben, C. M. \& Graeff, F. G. (2007). Elevated mazes as animal models of anxiety: Effects of serotonergic agents. Anais de Academia Brasileira de Ciências, 79, 71-85.

Pum, M. E., Huston, J. P. \& Muller, C. P. (2009). The role of cortical serotonin in anxiety and locomotor activity in Wistar rats. Behavioral Neuroscience, 123, 449-454.

Salchner, P. \& Singewald, N. (2002). Neuroanatomical substrates involved in the anxiogenic-like effect of acute fluoxetine treatment. Neuropharmacology, 43, 1238-1248. 
Schwabe, L., Bohringer, A. \& Wolf, O. T. (2009). Stress disrupts context-dependent memory. Learning and Memory, 16, 110-113.

Schwabe, L. \& Wolf, O. T. (2010). Learning under stress impairs memory formation. Neurobiology of Learning and Memory, 93, 183-188.

Silva, R. C. \& Brandao, M. L. (2000). Acute and chronic effects of gepirone and fluoxetine in rats tested in the elevated plus-maze: An ethological analysis. Pharmacology, Biochemistry and Behavior, 65, 209-216.

Silva, R. C., Gargaro, A. C. \& Brandao, M. L. (2004). Differential regulation of the expression of contextual freezing and fear-potentiated startle by 5-HT mechanisms of the median raphe nucleus. Behavioural Brain Research, 151, 93-101.

Sodero, A. O., Valdomero, A., Cuadra, G. R., Ramírez, O. A. \& Orsingher, O. A. (2004). Locus coeruleus activity in perinatally protein-deprived rats: Effects of fluoxetine administration. European Journal of Pharmacology, 503, 35-42.

Strobel, A., Dreisbach, G., Muller, J., Goschke, T., Brocke, B. \& Lesch, K. P. (2007). Genetic variation of serotonin function and cognitive control. Journal of Cognitive Neuroscience, 19, 1923-1931.
Strong, P. V., Greenwood, B. N. \& Fleshner, M. (2009). The effects of the selective 5-HT(2C) receptor antagonist SB 242084 on learned helplessness in male Fischer 344 rats. Psychopharmacology (Berl), 203, 665-675.

Taylor, S. \& Stein, M. B. (2006). The future of selective serotonin reuptake inhibitors (SSRIs) in psychiatric treatment. Medical Hypotheses, 66, 14-21.

Terry, A. V., Jr., Buccafusco, J. J. \& Wilson, C. (2008). Cognitive dysfunction in neuropsychiatric disorders: Selected serotonin receptor subtypes as therapeutic targets. Behavioural Brain Research, 195, 30-38.

Thomasius, R., Zapletalova, P., Petersen, K., Buchert, R., Andresen, B., Wartberg, L. et al. (2006). Mood, cognition and serotonin transporter availability in current and former ecstasy (MDMA) users: The longitudinal perspective. Journal of Psychopharmacology, 20, 211-225.

Viana, M. B., Zangrossi, H., Jr. \& Onusic, G. M. (2008). 5-HT1A receptors of the lateral septum regulate inhibitory avoidance but not escape behavior in rats. Pharmacology, Biochemistry and Behavior, 89, 360-366 